\title{
Restoration of Humeral Bone Stock Two Years After Internal Fixation of a Periprosthetic Fracture with a Loose Stem
}

\author{
A Report of Two Cases \\ Christian Fang, FRCS, Chun-Hoi Yan, FRCS, Dennis Yee, FRCS, Tak-Wing Lau, FRCS, Tak-Man Wong, FRCS, \\ and Frankie Leung, FRCS
}

Investigation performed at the Department of Orthopaedics and Traumatology, University of Hong Kong Queen Mary Hospital, Hong Kong

\begin{abstract}
Case: We describe 2 patients who sustained a periprosthetic humeral fracture with a loosened long-stemmed CoonradMorrey total elbow prosthesis. As noted in the literature, the success rate for a major revision with use of strut grafts is around $70 \%$; therefore, both cases were managed without revision of the prosthesis. A submuscular locking plate was placed following typical fracture fixation principles. Screws that interfered with the humeral stem and the distal flange stabilized both the distal fragment and the humeral stem.
\end{abstract}

Conclusion: At 2 years postoperatively, both fractures had healed, with increased endosteal bone stock. In each case, the prosthesis was successfully salvaged, and radiographic reconstitution of the implant-bone interface was noted after 2 years.

$\mathrm{P}$ eriprosthetic humeral fractures after total elbow replacement are challenging to manage when there is implant loosening, poor bone stock, and osteopenia. Surgeons tend to feel that a loose stem needs to be addressed when operating on a periprosthetic fracture. A few reports have described successful management with revision of the stem to a longer one, recementation, placement of a strut allograft, and circumferential cable reinforcement, with a reported success rate of approximately $70 \% \%^{1,2}$. Alternative techniques with impaction bone-grafting have been unsuccessful ${ }^{3}$ as a result of insufficient torsional control ${ }^{4}$. We report 2 cases of humeral fracture around a loosened long-stem elbow prosthesis. We used only angle-stable fixation; we did not use strut grafts or cement, and we did not revise the prosthesis. In both patients, rotationally stable osteosynthesis with re-established stability was evident after 2 years.

The patients were informed that data concerning their cases would be submitted for publication, and they both provided consent.

\section{Case Report}

CASE 1. After a fall on level ground, a 93-year-old woman $\checkmark$ presented with a closed periprosthetic fracture of the left humerus (H-II2 type according to the Mayo Classification of
Periprosthetic Fractures of the Elbow ${ }^{5}$. She had received a Coonrad-Morrey total elbow replacement with a 6-in (15.24-cm) humeral component 10 years prior for a comminuted intraarticular fracture. She had known that she had a loosened humeral stem for the past 2 years, but she had declined revision surgery because the symptoms remained minimal. At the time of presentation after the fall, there was no neurovascular injury. Blood work showed a normal C-reactive protein level and a normal erythrocyte sedimentation rate. She also had a concomitant ipsilateral impacted femoral neck fracture. She agreed to fixation of both the humeral and femoral fractures, but declined revision of the loosened elbow prosthesis. The loosened stem remained contained within the intact humeral cortex, with no substantial cortical bone loss.

Surgery was performed 2 days after the injury under general anesthesia. First, the femoral neck fracture was fixed with 3 cannulated screws with the patient on a traction table. She was then transferred to a beach-chair position. For the humeral fixation, a deltoid-splitting approach was used proximally, and a lateral approach was used distally with identification of the radial nerve. A 199-mm-long proximal humeral periarticular locking compression plate (DePuy Synthes) was slid in submuscularly. The fracture was reduced with the use

Disclosure: The authors indicated that no external funding was received for any aspect of this work. On the Disclosure of Potential Conflicts of Interest forms, which are provided with the online version of the article, one or more of the authors checked "yes" to indicate that the author had a relevant financial relationship in the biomedical arena outside the submitted work. 
JBJS Case Connector Volume 7 • Number $1 \cdot$ March 8,2017
Restoration of Humeral Bone Stock After internal Fixation of a Periprosthetic Fracture of bone-holding clamps. The prosthesis was loose, with a few millimeters of toggling possible at the fracture. The stem was jammed between multiple screws and the intact posterior humeral cortex. Three 3.5-mm locking screws and 2 cortical screws were placed in the distal fragment just anterior to the humeral stem, with 2 in the window behind the anterior flange and the stem, which interfered with the prosthesis. Proximally, there were 6 locking screws and 2 cortical screws. After fixation, the prosthesis became immobile as noted with fluoroscopic screening.

Postoperatively, free mobilization of the left shoulder and elbow was allowed in order to facilitate full weight-bearing exercise on the operatively treated hip with a walker. At 3 months, the fracture had healed, and the patient had elbow range of motion from $5^{\circ}$ to $140^{\circ}$. She was followed for 2 years and remained painfree with nearly full range of motion. Radiographs demonstrated serial improvement of the endosteal and periosteal bone stock from the fracture callus, and there was prosthetic stem stability (Fig. 1).

CASE 2. A 62-year-old woman with rheumatoid arthritis had received a right-sided total elbow replacement 2 years prior. One year after the initial surgery, she had experienced aseptic loosening of the humeral component. The primary humeral stem was revised to a 6-in $(15.24-\mathrm{cm})$ humeral component and was cemented and reinforced with a strut allograft. This operation had been complicated because of radial nerve palsy. A year later, she returned with a periprosthetic fracture of the humerus and loosening of the stem following a minor injury during physiotherapy. She was on $2 \mathrm{mg}$ of prednisolone therapy daily. Her history included replacement of the right knee, fusion of $\mathrm{C} 1-\mathrm{C} 2$, instrumented fixation of a T12 vertebral collapse, and plating of a fracture of the clavicle. The inflammatory markers were normal.

The fracture was a borderline H-II1 (stable stem)/H-II2 (unstable stem) type with a radiolucent line surrounding $80 \%$ of the implant. Integration of the strut graft was not yet complete. We decided to bridge the fracture without stem revision since the distal bone-implant interface appeared relatively stable. A deltoid-splitting approach was used proximally, and a lateral approach was used distally with exploration of the radial nerve. The nerve was suspected to have suffered thermal injury from previously extruded cement. A cortical defect that was threequarters of the humeral circumference in size was present at the fracture. The prosthesis was mobile, but only to a minor degree. Cement around the fracture was removed, and the bone was shortened by $2 \mathrm{~cm}$ to improve contact. Iliac crest cancellous autograft was placed. A 196-mm-long PHILOS plate (DePuy Synthes) was used to span the entire humerus; multiple screws and cerclage wiring were placed in a manner similar to that described in Case 1. The patient was allowed gentle elbow and shoulder mobilization exercises immediately after surgery.

At 6 weeks postoperatively, the patient had an elbow arc ranging from $20^{\circ}$ to $100^{\circ}$. Fracture union was evident at 3 months. The patient received a flexor-to-extensor tendon transfer 1 year later for a wrist drop. At 2 years postoperatively, there was remodeling of the bone at the fracture site and the implant was stable (Fig. 2). She had pain-free range of motion from $10^{\circ}$ to $110^{\circ}$.

\section{Discussion}

7 he most common reasons for revision after total elbow replacement surgery are aseptic loosening ${ }^{6-9}$, bushing wear, and periprosthetic fracture $e^{9,10}$. For a patient with a periprosthetic femoral fracture and a potential loose hip or knee prosthesis, revision is clearly recommended. However, with a periprosthetic humeral fracture, there is very limited evidence to define the standard of care. Recommended by many ${ }^{2,11,12}$, revision and strut allograft augmentation is at best based on 2 series of patients



Fig. 1

Radiographs (preoperatively and at 3, 12, and 24 months) demonstrating serial remodeling of bone and replenishment of bone stock from callus at

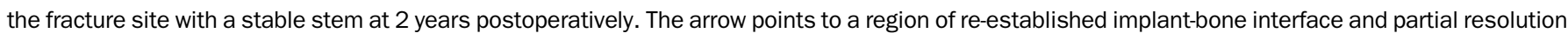
of the previously continuous radiolucent line near the fracture site (Case 1). 


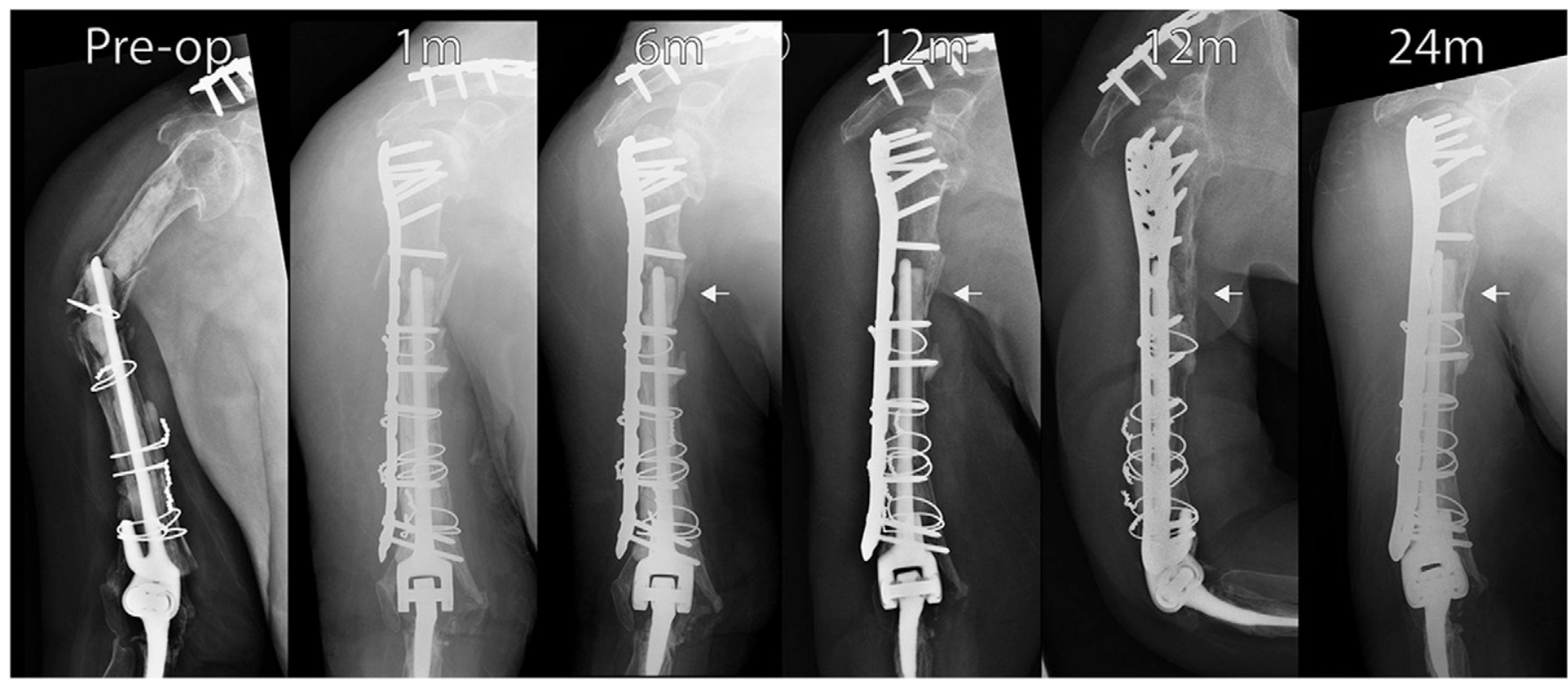

Fig. 2

Radiographs (preoperatively and at 1, 6, 12, and 24 months) demonstrating serial remodeling of bone and replenishment of bone stock from callus at the fracture site and a stable stem at 2 years postoperatively. The arrow points to a region of reestablished cement-bone interface and complete disappearance of the radiolucent line (Case 2).

reported by Sanchez-Sotelo et al. ${ }^{1}$ and Trompter and Gupta ${ }^{2}$, where 4 of 14 patients had nonunion, persistent loosening, or recurrent fractures.

The surgical plan for periprosthetic fractures in the humerus must take implant stability, fracture configuration, and bone stock into account. Angle-stable plate osteosynthesis with cables or cerclage wires is a successful treatment method for patients without prosthetic loosening ${ }^{13,14}$. Component revision is generally thought to be necessary whenever there is prosthetic loosening ${ }^{12}$. Strut graft augmentation along with plating is recommended when there is insufficient bone stock ${ }^{11,15}$. A tumor prosthesis serves as a last resort when there is a very large segmental bone defect that cannot be reconstructed ${ }^{16}$.

While the management of a periprosthetic fracture in the humerus shares certain similarities with a periprosthetic fracture in the femur, the importance of rotational stability must not be overlooked. Unlike the femur, which mainly supports the body through axial stiffness, the humerus must resist considerable torsional stress transferred from the forearm under physiological loads. Because a stemmed humeral implant has an inherent moment arm disadvantage at the center, its torsional stability is prone to be rapidly compromised by cement interface failure and endosteal bone erosion ${ }^{4}$.

To establish rotational stability of loose stems following total elbow replacement, Papadonikolakis et al. ${ }^{4}$ suggested impacting a triangular piece of intramedullary strut graft between the endosteal bone and the implant at revision. Alternatively, we used screws to establish torsional control in a manner similar to interlocking intramedullary nails ${ }^{17}$. The Coonrad-Morrey total elbow replacement humeral component features a triangularprofiled stem and a distal anterior flange designed to provide rotational stability. Based on these design features, screws are placed into the area between the distal flange and the stem, and the stem can be sandwiched between multiple interfering screws to give considerable torsional control (Fig. 3).

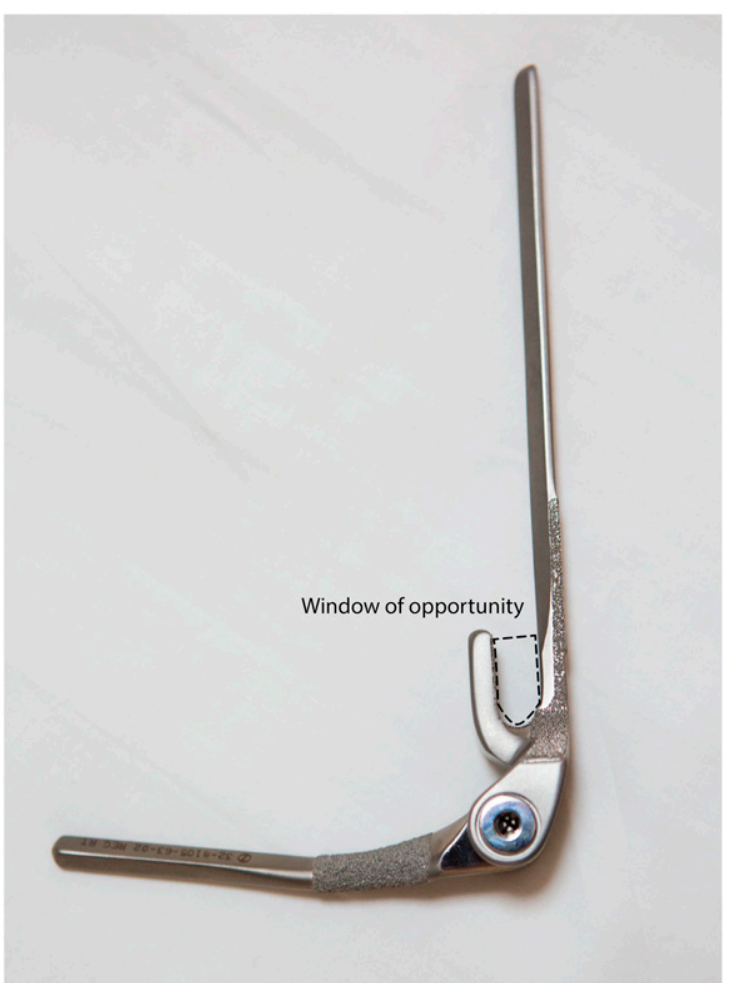

Fig. 3

The "window of opportunity" is located behind the flange of the humeral component, where interfering screws can provide torsional control. 
The technique described here should only be applied in situations with aseptic loosening without large segmental or uncontained cortical defects. Minor degrees of axial pistoning or rotational movements of the prosthesis are acceptable, but the axial position of the stem must be correct without varus, valgus, flexion, or extension malalignment or instability. The cortex of the humerus must be intact in order to contain the prosthesis if interfering screws are to be placed on only 1 side. Sufficient bone stock (or cement) must be available at the area behind the distal flange for screw purchase. The radial nerve requires routine identification since implants are placed laterally, and the need for cerclage wires requires a larger distal incision. For longer screws to be placed, the plate must be accurately positioned off-center with the use of Kirschner wires; this should be confirmed with fluoroscopy. The drill-bit is at risk of breakage, and excessive drilling may lead to cement cracks that compromise prosthetic stability. In high-demand or heavy patients, screws interfering with the humeral prosthesis alone may not be sufficient, leading to metallosis, eventual screw breakage, and recurrent loosening.

Although treating a diaphyseal humeral fracture with a potentially loose prosthesis by plating alone may appear unconventional, it has certain appeal, especially with lowerdemand patients. As illustrated by the radiographs in the 2 cases described herein, replenishment of bone stock around the prosthesis can occur from callus. We hypothesize that this is only possible when internal fixation is applied in a way that immediately establishes torsional stability. Periosteal stripping should be minimized, and autogenous cancellous bonegrafting may contribute to success. We suggest that additional investigation regarding this technique is necessary, preferably with specially designed implants that offer 2 rows of anglestable screws.

This simplified technique is a feasible alternative to major revision surgery. Angle-stable plating with multiple interfering screws immediately establishes rotational stability. Both of our patients healed with recovery times similar to uncomplicated humeral shaft fractures. Long implants spanning the entire length should protect the humerus from an additional fracture. In both cases, the callus appears to have replenished endosteal and periosteal bone stock inside and outside of the fracture. At the 2-year follow-up, both of the originally loose prostheses seemed to have regained stability without any problems. If the stems had become loose with a healed fracture, contemporary revision techniques also would be relatively straightforward.

Christian Fang, FRCS ${ }^{1}$

Chun-Hoi Yan, FRCS ${ }^{1}$

Dennis Yee, FRCS ${ }^{1}$

Tak-Wing Lau, FRCS ${ }^{1}$

Tak-Man Wong, FRCS ${ }^{1,2}$

Frankie Leung, FRCS ${ }^{1,2}$

${ }^{1}$ Department of Orthopaedics and Traumatology, University of Hong Kong Queen Mary Hospital, Hong Kong

${ }^{2}$ Shenzhen Key Laboratory for Innovative Technology in Orthopaedic Trauma, University of Hong Kong Shenzhen Hospital, Shenzhen, People's Republic of China

E-mail address for C. Fang: fangcx@gmail.com

\section{References}

1. Sanchez-Sotelo J, O'Driscoll S, Morrey BF. Periprosthetic humeral fractures after total elbow arthroplasty: treatment with implant revision and strut allograft augmentation. J Bone Joint Surg Am. 2002 Sep;84(9):1642-50.

2. Trompeter AJ, Gupta RR. The management of complex periprosthetic humeral fractures: a case series of strut allograft augmentation, and a review of the literature. Strategies Trauma Limb Reconstr. 2013 Apr;8(1):43-51. Epub 2013 Feb 28.

3. Loebenberg MI, Adams R, O'Driscoll SW, Morrey BF. Impaction grafting in revision total elbow arthroplasty. J Bone Joint Surg Am. 2005 Jan;87(1):99-106. Epub 2005 Jan 7. 4. Papadonikolakis A, McKenna M, Warme WJ, Matsen FA. Intramedullary fibular and impaction allografting in revision total elbow arthroplasty with endosteal deficiency. Tech Hand Up Extrem Surg. 2012 Mar;16(1):5-11. Epub 2012 Mar 1.

5. O'Driscoll SW, Morrey BF. Periprosthetic fractures about the elbow. Orthop Clin North Am. 1999 Apr;30(2):319-25. Epub 1999 Apr 10.

6. Voloshin I, Schippert DW, Kakar S, Kaye EK, Morrey BF. Complications of total elbow replacement: a systematic review. J Shoulder Elbow Surg. 2011 Jan;20(1):158-68.

7. Goldberg SH, Urban RM, Jacobs JJ, King GJ, O'Driscoll SW, Cohen MS. Modes of wear after semiconstrained total elbow arthroplasty. J Bone Joint Surg Am. 2008 Mar:90(3):609-19. Epub 2008 Mar 4.

8. Skyttä ET, Eskelinen A, Paavolainen P, Ikävalko M, Remes V. Total elbow arthroplasty in rheumatoid arthritis: a population-based study from the Finnish Arthroplasty Register. Acta Orthop. 2009 Aug;80(4):472-7. Epub 2009 Jun 30. 9. Aldridge JM 3rd, Lightdale NR, Mallon WJ, Coonrad RW. Total elbow arthroplasty with the Coonrad/Coonrad-Morrey prosthesis. A 10- to 31-year survival analysis. J Bone Joint Surg Br. 2006 Apr;88(4):509-14.
10. Throckmorton $T$, Zarkadas $P$, Sanchez-Sotelo J, Morrey $B$. Failure patterns after linked semiconstrained total elbow arthroplasty for posttraumatic arthritis. J Bone Joint Surg Am. 2010 Jun;92(6):1432-41. Epub 2010 Jun 3.

11. Kieser DC, Taylor F, Ball CM. The allograft sleeve: a case report of the surgical management of an interprosthetic fracture between a shoulder and elbow joint replacement. J Shoulder Elbow Surg. 2011 Apr;20(3):e4-9.

12. Dehghan N, Chehade M, McKee MD. Current perspectives in the treatment of periprosthetic upper extremity fractures. J Orthop Trauma. 2011 Jun;25(Suppl 2): S71-6.

13. Kent ME, Sinopidis C, Brown DJ, Frostick SP. The locking compression plate in periprosthetic humeral fractures A review of two cases. Injury. 2005 Oct;36 (10):1241-5. Epub 2005 Jun 27.

14. Mavrogenis AF, Angelini A, Guerra E, Rotini R. Humeral fracture between a total elbow and total shoulder arthroplasty. Orthopedics. 2011 Apr 11;34(4):315.

15. Martinez AA, Calvo A, Cuenca J, Herrera A. Internal fixation and strut allograft augmentation for periprosthetic humeral fractures. J Orthop Surg (Hong Kong). 2011 Aug;19(2):191-3.

16. Funovics PT, Schuh R, Adams SB Jr, Sabeti-Aschraf M, Dominkus M, Kotz RI. Modular prosthetic reconstruction of major bone defects of the distal end of the humerus. J Bone Joint Surg Am. 2011 Jun 1;93(11):1064-74.

17. Blum J, Karagül G, Sternstein W, Rommens PM. Bending and torsional stiffness in cadaver humeri fixed with a self-locking expandable or interlocking nail system: a mechanical study. J Orthop Trauma. 2005 Sep;19(8):535-42. Epub 2005 Aug 25. 